\title{
Functional neuroimaging of traumatic brain injury: advances and clinical utility
}

This article was published in the following Dove Press journal:

Neuropsychiatric Disease and Treatment

15 September 2015

Number of times this article has been viewed

\author{
Andrei Irimia \\ John Darrell Van Horn \\ USC Stevens Neuroimaging and \\ Informatics Institute, Keck School \\ of Medicine, University of Southern \\ California, Los Angeles, CA, USA
}

\begin{abstract}
Functional deficits due to traumatic brain injury (TBI) can have significant and enduring consequences upon patients' life quality and expectancy. Although functional neuroimaging is essential for understanding TBI pathophysiology, an insufficient amount of effort has been dedicated to the task of translating functional neuroimaging findings into information with clinical utility. The purpose of this review is to summarize the use of functional neuroimaging techniques - especially functional magnetic resonance imaging, diffusion tensor imaging, positron emission tomography, magnetic resonance spectroscopy, and electroencephalography for advancing current knowledge of TBI-related brain dysfunction and for improving the rehabilitation of TBI patients. We focus on seven core areas of functional deficits, namely consciousness, motor function, attention, memory, higher cognition, personality, and affect, and, for each of these, we summarize recent findings from neuroimaging studies which have provided substantial insight into brain function changes due to TBI. Recommendations are also provided to aid in setting the direction of future neuroimaging research and for understanding brain function changes after TBI.
\end{abstract}

Keywords: cognitive decline, personality change, magnetic resonance imaging, diffusion tensor imaging

\section{Introduction}

Traumatic brain injury (TBI) is a clinical condition which can have substantial and long-lasting effects not only upon brain structure, but also upon motor ability, attention, memory, higher cognition, personality, and affect. Due to the wide range of mechanisms whereby brain injury can be inflicted, the etiology of this condition is varied and often complex, as is the host of potential effects that TBI can have upon life quality and expectancy. Monitoring primary TBI evolution and optimizing clinical care in response to secondary injury are often challenging due to the structural heterogeneity of brain insults across patients, as well as the multifaceted nature of metabolic, neurochemical, and electrophysiological responses prompted by neural injury. Currently, a wide range of experimental techniques are used to probe brain function changes which occur after TBI. Because of the intimate relationship between brain structure and function, a complementary relationship exists between structural and functional neuroimaging, and a large proportion of functional neuroimaging studies of TBI involve the use of structural neuroimaging as well. In a previous review, ${ }^{1}$ we highlighted the importance of structural neuroimaging in identifying neuroimaging biomarkers with predictive clinical value, and discussed the role of magnetic resonance imaging (MRI) and diffusion tensor imaging (DTI) in formulating next-generation approaches for the treatment of TBI. Combining imaging modalities can lead to substantial insights into brain structure and function, which cannot be obtained using only one technique by itself. This is the
Correspondence: John Darrell Van Horn USC Stevens Neuroimaging and Informatics Institute, Keck School of Medicine, University of Southern California, 200I North Soto Street, SSBI-102, Los Angeles, CA 90032, USA

$\mathrm{Tel}+\mathrm{I} 3234427246$

Fax +I 3234420137

Email jvanhorn@usc.edu 
case to such an extent ${ }^{2}$ that multimodal neuroimaging using an ever-increasing number of imaging methods has been a pervasive trend since at least the mid-1990s. Since then, the number of techniques for brain imaging has also increased. For example, a thorough review by the Pediatric TBI Neuroimaging Workgroup 3 identified no fewer than 15 structural or functional macroscopic neuroimaging modalities which have been approved for use in human subjects. These include not only widely available techniques such as MRI, functional MRI (fMRI), and DTI, but also MRI sequences which are tailored to TBI, including susceptibility weighted imaging, fluid-attenuated inversion recovery, and gradient-recalled echo MRI. Functional modalities also include magnetic resonance spectroscopy (MRS), single-photon emission tomography, positron emission tomography (PET), electroencephalography (EEG), magnetoencephalography (MEG), and multispectral imaging. From among these, fMRI, PET, and EEG are the most commonly available techniques in both clinical and research settings.

In the present review, we explore the importance of functional neuroimaging techniques - including fMRI, PET, MRS, and EEG - for advancing the state of the art in TBI patient clinical care and rehabilitation. Specifically, we highlight the clinical utility of functional neuroimaging in understanding TBI dysfunction and in advancing clinical care by discussing, in turn, several functional domains which are typically affected by TBI, namely consciousness, motor function, attention, memory, higher cognition, personality, and affect. For each of these categories, we summarize important findings of functional neuroimaging studies, which were published in or after 2010 and which have contributed to our understanding of brain function changes which occur after TBI. We conclude by providing recommendations that can aid in setting the direction of future neuroimaging research for understanding brain function changes after this type of brain injury.

\section{Consciousness}

There has been renewed interest, throughout the past decade, in the use of functional neuroimaging to distinguish between patients in the vegetative state versus individuals who are in a minimally conscious state or who exhibit locked-in syndrome. Garcia-Panach et $\mathrm{al}^{4}$ for example, used fluorodeoxyglucose (FDG)-PET to study correlations and differences in glucose metabolism between the thalamus and cortical structures in 49 severe TBI patients and ten healthy control subjects, including 17 patients in either vegetative or minimally conscious state. Because of connections which link the thalamus to the ascending reticular system and which regulate sleep-wake transitions, the thalamus plays a central role in the maintenance of awareness after TBI, in conjunction with the precuneus and frontal lobe areas. ${ }^{5}$ With this knowledge in mind, Garcia-Panach et $\mathrm{al}^{4}$ used voxel-based morphometry to test the hypothesis that functional connectivity between these structures is involved in TBI-related neurological and functional outcome. A general linear model framework was used to compare glucose metabolism levels in TBI patients to those in Huntington's chorea and to those with posttraumatic amnesia. The most significant statistical differences in glucose utilization were found between healthy control subjects and the minimally-conscious/vegetative-state group, where the precuneus and temporal cortex showed the largest effect sizes across the brain regions inspected. TBI severity was found to be associated significantly with glucose hypometabolism, and a significant correlation was found between cortico-thalamo-cortical metabolism and functional outcome. Although the authors did not compare vegetative-state patients with minimally-conscious patients, posttraumatic hypometabolism was found to resolve in tandem with improvements in neurological outcome. Metabolic abnormalities were found to be smallest in the posttraumatic amnesia group, which may suggest that FDG-PET can be useful for distinguishing between vegetative-state and minimally-conscious patients, although further research focused on metabolic differences between these groups is needed. Of particular interest is determining whether thalamic hypometabolism is due to direct focal lesions, damage in the white matter resulting from diffuse axonal injury (DAI), or remote axonal disconnection and deafferentation. Though the latter mechanism appears to be best supported by evidence presented by Garcia-Panach et $\mathrm{al}^{4}$ future multimodal studies should attempt to combine functional techniques - such as FDG-PET and fMRI - with techniques for mapping structural connectivity - such as DTI and diffusion spectrum imaging to identify the neuroanatomical changes which modulate thalamic and cortical hypometabolism in TBI patients.

In a fascinating review of famous patients exhibiting locked-in syndrome, Bruno et $\mathrm{al}^{6}$ proposed that patients who show nonbehavioral evidence of consciousness or communication, which is only measurable via paraclinical testing (eg, via fMRI, PET, or EEG), should be considered to exhibit a functional locked-in syndrome. This suggestion finds support in the groundbreaking fMRI study of Owen and Coleman, ${ }^{7}$ who asked patients in vegetative state to perform mental imagery tasks and then found robust activation in the supplementary motor area or in the parahippocampal gyrus, 
depending on the task the volunteers had been instructed to perform. In studies by Schnakers et al ${ }^{8,9}$ electromyography recordings even helped in detecting consciousness in a patient with a rare case of complete locked-in syndrome, who had been behaviorally diagnosed as comatose. These and other studies indicate that functional neuroimaging holds considerable promise in the effort to classify TBI patients as being in either vegetative, minimally-conscious, or locked-in state, and to formulate clinical treatments and strategies for rehabilitation based on measures of event-related blood flow changes, glucose utilization, and electrophysiological activity.

\section{Motor function}

Motor coordination and acuity are very frequently affected by TBI, and recovery of motor abilities can require a substantial amount of physical therapy in brain injury patients. This is the case partly because the coordination and successful completion of motor actions frequently rely upon the information-processing speed of patients as well as upon their ability to sustain attention and to switch between tasks. ${ }^{10}$ Because the latter three brain functions are very often affected by TBI, this implies that the neural mechanisms underlying motor control are frequently affected by injury. When this is the case, patients' ability to lead their lives without frequent assistance from caregivers may be compromised for prolonged periods of time if the deficits are sufficiently severe. Even in cases of moderate damage to motor function, the ability to perform tasks which require complex motor control (eg, walking, writing, typing, driving) may be compromised to the extent that the resumption of routine activities is quite problematic. Because of such challenges which affect a substantial proportion of TBI survivors, much effort has been devoted to studying the mechanisms whereby primary and secondary injuries to the brain lead to the deterioration of motor function.

Important recent research has been performed to identify which functional networks involved in movement are degraded by TBI and how. Many brain functions involved in motor control - eg, sustained attention, task switching, and information-processing speed - are localized in distinct brain regions and additionally involve neuronal networks which are spatially distributed. Thus, the scenario according to which highly focal lesions cause brain-wide disruptions to motor function is not compatible with the distributed nature of these networks. With such considerations in mind, Kasahara et $\mathrm{al}^{11}$ posited that damage to abilities which affect motor function may in fact be partially due to DAI. These authors investigated how TBI-induced damage affects brain networks far from primary lesion sites in 12 TBI patients and in nine healthy control subjects while they performed a finger-thumb opposition task. A psychophysiological interaction analysis which examined the integrity of functional connectivity across brain sites was used in conjunction with fMRI to determine how blood-oxygen-level dependent (BOLD) signals change differently in TBI patients compared to healthy control subjects. In the TBI patients examined, this led to the finding that the primary motor cortex (M1), cerebellum, and supplementary motor area exhibit reduced interhemispheric interactions as well as abnormal ipsilateral interactions with the supramarginal gyrus. It is perhaps not a coincidence that the supramarginal gyrus and other structures of the parietal lobe have been involved in the default mode network (DMN) of the brain, ${ }^{12}$ in its connectivity scaffold, ${ }^{13,14}$ in the interpretation of sensory data, ${ }^{15}$ and in perceiving space and limb location. ${ }^{16}$ The poor interaction observed between the cerebellum and M1 resonates with the possibility that DAI may be partly responsible for the loss of motor acuity observed in TBI patients, whereas the functional deterioration associated with the supramarginal gyrus is consistent with previous hypotheses which assign a causal relationship between DMN damage, on the one hand, and loss of motor function after TBI, on the other hand. Incidentally, structural neuroimaging methods previously allowed us to quantify substantial decreases in cerebellar volume in severe TBI patients 6 months after injury, ${ }^{17}$ which highlights the challenge of patient rehabilitation in the face of such brain tissue losses difficult to reverse. Based on evidence provided by their functional neuroimaging study, Kasahara et al concluded that the abnormal activation patterns observed in their TBI patients are probably due to the massive reorganization of motor activation networks after TBI. ${ }^{11,23}$

Intimately related to motor acuity is task-switching ability. Leunissen et $\mathrm{al}^{18}$ used fMRI in conjunction with DTI tractography to study TBI brain function changes associated with motor switching performance in 23 young adults with moderate-to-severe TBI. In their study, visual cues were used to prompt the initiation and termination of spatially and temporally coupled bimanual circular movements. Specifically, the right hand was cued to either switch or to continue its circling direction movement, and the time to initiate the switch (switch response time) was measured in both TBI patients and control subjects. As expected, the study group was found to exhibit longer switch response times as well as a higher incidence of complete contralateral movement disruptions. Although both groups activated their basal 
ganglia, supplementary motor cortex, and bilateral inferior frontal cortex, TBI patients showed increased activation of the presupplementary motor area and left inferior frontal cortex, as well as underactivation of the subthalamic nucleus region. The authors found that the differences between the control group and the study group were due to altered white matter circuitry in TBI between brain regions involved in the task, suggesting that damage to these key pathways causes TBI patients to perform more poorly.

Of substantial importance in motor function is interhemispheric coordination, which can be conveniently quantified using an interhemispheric transfer time measure. Babikian et $\mathrm{al}^{19}$ for example, studied DAI secondary to TBI and attempted to characterize the relationship between structural damage (DAI) and metabolic status in the anterior and posterior corpus callosum. These authors acquired MRS, fMRI, and DTI volumes from a total of 18 patients, who were dichotomized into two groups, namely postacute (5 months postinjury) and chronic (15 months postinjury). Neurobehavioral measures such as bimanual coordination and the interhemispheric transfer time were recorded. Creatine - a biomarker of energy metabolism - was not found to differ across groups, though choline - a biomarker of membrane degeneration and inflammation - was elevated acutely, but not chronically. $N$-Acetyl aspartate is a surrogate biomarker of neuronal/axonal integrity, which was found to be lower than normal in the acute stage of TBI, although closer to normal levels in the chronic TBI group. Interestingly, $N$-acetyl aspartate in the posterior corpus callosum was positively correlated with neurocognitive function and negatively correlated with the interhemispheric transfer time. The findings of the study highlighted the usefulness of studying, in addition to changes in the BOLD response, the temporal evolution of metabolic biomarkers when attempting to identify the mechanisms underlying motor deficits due to TBI. Because the relationship between the BOLD response and glucose utilization is nonlinear and complex, ${ }^{20}$ obtaining measures of metabolic dynamics such as FDG utilization, creatine, choline, $N$-acetyl aspartate, and serum measurements obtained via microdialysis or otherwise should be prioritized in TBI research studies.

\section{Cognitive function}

Although posttraumatic amnesia and other memory problems are frequent consequences of $\mathrm{TBI},{ }^{21}$ very little is currently known about how brain function differs in the posttraumatic amnesia stage of TBI compared to the stage where recovery from amnesia has occurred. Garcia-Panach et $\mathrm{al}^{4}$ lamented this lack of knowledge and suggested that future studies should strive to provide substantial insight into how functional connectivity - particularly between the frontal and the temporal lobe - modulates recovery from amnesia. These authors implemented an FDG-PET study which included 12 patients with posttraumatic amnesia and 20 patients who had recovered from it, all of whom were found to exhibit metabolic dysfunction in subfrontal and medial temporal areas. Given that memory consolidation and retrieval are two prominent functions of medial temporal lobe areas, ${ }^{22}$ this finding is intuitive. Nevertheless, TBI is more likely to affect temporal and orbitofrontal areas due to the anatomy and positioning of the brain within the skull in the context of TBI mechanics. For this reason, it is difficult to discern whether these areas exhibit metabolic dysfunction because of their involvement in memory processes or rather because TBI physics implies that these areas are injured more often.

Despite recent neuroimaging findings on which brain regions are involved in posttraumatic amnesia, the mechanisms responsible for the relationship between its duration and widespread brain dysfunction remain unknown. As in the case of motor function, however, both metabolic and blood oxygenation deficits in the parietal lobe have been implicated in abnormal memory encoding and retrieval. Kasahara et $\mathrm{a}^{23}$ used fMRI during an n-back working memory task in nine chronic TBI patients and nine matched controls to investigate the impact of injury upon functional brain networks which mediate working memory. At high working memory loads, as expected, TBI patients made a larger percentage of errors, with significant reductions in the activation of the left inferior parietal gyrus and increases in activity within the right inferior frontal gyrus. Psychophysiological interaction analysis further indicated that functional connectivity between these two structures was compromised in TBI patients, suggesting that abnormal interaction between the areas may underlie the observed working memory deficits and abnormal brain activation patterns of TBI patients.

Some TBI studies have identified overlaps of abnormal activity between working memory networks and the DMN, suggesting that DMN function is altered in patients who perform poorly on working memory tasks. Palacios et $\mathrm{a}^{24}$ for instance, implemented an fMRI n-back task experiment and acquired DTI volumes from 19 chronic TBI patients with evidence of axonal injury, as well as from an equal number of matched control subjects. After identifying both the working memory network and the DMN of each patient, the authors found correlations between the fractional anisotropy of a number of white matter tracts involved in functional 
activation, on the one hand, and the functional activation patterns of these fasciculi, on the other hand. By combining both structural and functional neuroimaging data, the researchers were able to describe structural brain changes which lead to functional network alterations and to lower working memory performance in the chronic TBI group.

Functional neuroimaging investigations which explore the recovery of memory acuity after TBI are few. In one such study, Kim et $\mathrm{al}^{25}$ used a perfusion fMRI approach which dissociates resting- and task-related cerebral blood flow changes to investigate the neural correlates of cognitive dysfunction and recovery after TBI. Perfusion fMRI using arterial spin labeling was employed by the authors to investigate cerebral blood flow both during the resting state as well as during a working memory task. A total of 21 TBI patients and 18 demographically matched healthy control subjects completed a sustained visual attention tasks as well as a 2-back working memory task. The TBI patients exhibited poorer behavioral performance on both tasks, with task-induced cerebral blood flow changes being localized to the superior occipital cortex bilaterally as well as to the left superior temporal cortex. Control subjects were found to deactivate these areas during task performance, but TBI participants were found to activate these areas, with a disproportionate amount of hypoperfusion at rest. Distinct patterns of correlation between performance and task-related cerebral blood flow changes were identified between the study group and the control group. The conclusion of the study was that the different patterns of performance-activation correlation between TBI patients and healthy control subjects were consistent with the hypothesis that sensory and attentional modulation deficits due to TBI contribute to higher memory dysfunction in this patient group long after brain injury.

As in the case of memory function, interhemispheric coordination plays a central role in the modulation of attention and reaction time. Marquez de la Plata et $\mathrm{al}^{26}$ examined the functional connectivity of the hippocampus and of selected frontal lobe circuits in 24 patients with DAI as well as in 16 age- and sex-matched healthy volunteers. BOLD signals from the hippocampus, anterior cingulate cortex, and dorsolateral prefrontal cortex were recorded, and the functional connectivity between these regions and their contralateral counterparts was computed using a Pearson's correlation coefficient. As expected, TBI patients were found to exhibit lower interhemispheric connectivity for the hippocampus and the anterior cingulate cortex, and more limited recruitment of DMN components located in the dorsolateral prefrontal cortex. In another study by Scheibel et $\mathrm{al}^{27} \mathrm{fMRI}$ was used to investigate brain reactions to an event-related stimulus-response compatibility task in 15 subjects with mild, chronic, or blast-related TBI and to compare their activation patterns to those of matched controls. After controlling for reaction time, a between-group analysis found increased activation during stimulus-response incompatibility within the anterior cingulate gyrus, medial frontal cortex, and the occipital lobes. The study also found evidence for increased task-related activation following TBI as well as other functional changes associated with emotional symptoms.

Although functional neuroimaging studies have shed light upon how brain tissue metabolism and oxygenation are impaired when TBI patients perform memory tasks, further research is needed to understand how this information can be translated for clinical use at the bedside. For example, there are very few studies which explore how memory rehabilitation protocols affect brain structure or hemodynamic/ metabolic measures, and thus, it is currently very difficult to associate specific functional neuroimaging profiles with neuropsychological measures, which could aid in predicting and/or improving memory function. The studies reviewed here illustrate the intricate relationship between attention, response time, and memory, indicating that future studies should attempt to explore these three aspects of brain function in the same sample of TBI patients to avoid the potential presence of variables which confound efforts to compile meta-analyses. Such confounding variables include patient age, intellectual capacity, mechanism of injury, spatial injury profile, and DAI profile, all of which can pose substantial challenges when attempting to integrate scientific knowledge across multiple studies in a common frame of reference.

The deterioration of cognitive control abilities is among the most common sequelae of TBI, and recent functional neuroimaging studies have identified plausible mechanisms which may be responsible for the progressive loss and potentially slow recovery of cognitive control which occur after injury. Sponheim et $\mathrm{al}^{28}$ for example, used EEG and DTI to study cognitive deficits as well as disrupted coordination of brain activity in a group of nonimpact blast-related mild TBI patients who were soldiers returning from deployment to Afghanistan and Iraq. These researchers attempted to find whether neural communication between brain regions and coordination of neural function was associated with white matter integrity measures. Using a time-frequency method for measuring phase synchronization between EEG sensors, the authors found that TBI patients exhibited diminished EEG phase synchrony between frontolateral sites and contralateral brain regions, which suggested diminished 
interhemispheric coordination as a consequence of brain injury. These abnormal findings were also associated with diminished structural integrity of white matter tracts connecting the left anterior thalamic radiations and the forceps minor, including the anterior corpus callosum. Combat stress symptoms - such as posttraumatic stress disorder or clinical depression - and psychotropic medications were not found to be correlated significantly with the presence of diminished phase synchrony recorded using EEG. This may indicate that poor coordinates of frontal neural function after blast injury may be the consequence of damaged anterior white matter tracts in the TBI patient group studied. For a thorough review which summarizes the use of EEG to study cognitive control in TBI patients, the reader is referred to the excellent work of Duncan et $\mathrm{al}^{29}$ who analyzed brain event-related potentials as indices of functional pathophysiology in TBI survivors.

Studies have shown that the DMN is heavily involved in the modulation of cognitive control, ${ }^{30}$ and it is thus no surprise that abnormalities in the function of the former are also associated with failures of the latter. Because the activation of deep-brain structures is difficult to study using EEG or MEG, ${ }^{31,32} \mathrm{fMRI}$ and PET remain methods of choice for investigating the involvement of the midbrain in cognitive tasks which activate these structures, despite their relatively low temporal resolution compared to the former two methods. In an fMRI study, Palacios et $\mathrm{al}^{33}$ studied abnormalities in low-frequency fluctuations of BOLD signal amplitudes as well as resting-state connectivity in relationship to cognitive outcome in patients with severe DAI. The authors implemented independent component analysis, dual regression, and seed-based connectivity analysis to investigate functional connectivity within the DMN. They found that TBI patients had larger BOLD signal fluctuations in frontal areas, where fMRI signal variability was correlated with cognitive performance. Connectivity between the frontal lobe and parietal regions was found to be increased in TBI patients, and the fractional anisotropy of the cingulate fasciculus was correlated with increased frontal lobe connectivity within the DMNs of TBI patients. The authors suggested that loss of connectivity due to cingulum tract damage could be explained by compensatory increases in functional connectivity within frontal lobe portions of the DMN.

Aside from DMN studies, recent advances in connectomics and white matter tractography have allowed researchers to study the topological properties of brain networks and how these properties are affected by brain injury. For example, Pandit et $\mathrm{al}^{34}$ tested the hypothesis that brain networks involved in cognitive function may lose part of their small-world characteristics following TBI. These authors investigated resting-state functional connectivity using fMRI in a sample of $20 \mathrm{TBI}$ patients and 21 age-matched controls, and then used graph-theoretical methods and multivariate statistical models to infer relationships between changes in functional connectivity, on the one hand, and TBI-related white matter damage as measured using DTI, on the other hand. As expected, it was found that TBI leads to cognitive impairments associated with white matter damage as well as longer average path lengths and reduced network efficiency in TBI patients compared to control subjects. The posterior cingulate cortex - a brain network hub - was found to be particularly affected, likely due to the effect of DAI. Importantly, the study confirmed that functional networks in TBI patients shift away from small-world characteristics compared to those of healthy control subjects.

In an interesting study concerning the relationship between cognitive control, reaction time, and TBI effects, Wilde et $\mathrm{al}^{35}$ investigated the neural correlates of working memory using the Sternberg Item Recognition Task. Structural, functional, and diffusion MRI volumes were acquired from 40 children with moderate-to-severe TBI and 41 demographically matched control subjects who had orthopedic injuries. It was found that children with TBI had decreased cortical thickness compared to the control group, and additionally that left parietal lobe volume was negatively correlated to reaction time. The authors attributed their findings to the disruption of frontoparietal function during attention tasks, as quantified in both groups using DTI tractography. Decreased white matter integrity in the frontal lobes and in the cingulum bundle was associated with longer reaction times on the Sternberg Item Recognition Task, and the cingulum was found to emerge as a common structure related to performance after TBI.

Although cognitive control has traditionally been quantified using neuropsychological measurements, psychometric techniques cannot provide a mechanistic understanding of the causal links between injuries, their location, subsequent deterioration in structural connectivity, and functional deficits experienced by patients in their day-to-day lives. To accomplish this, studies are required to establish which structural and connectivity variables can be most confidently associated with changes in cognitive function after brain injury, and thereby to determine the causal chain which links neural impairment to high-level dysfunction. It is expected that functional neuroimaging will play an important role in this endeavor throughout the following decade, especially in studies of focal TBI, which offers otherwise unavailable 
opportunities to undertake case studies of localized human brain function.

\section{Personality and affect}

Although motor function, cognitive control, attention, and memory are often impaired after TBI, few factors impact the relationships between TBI survivors and society as much as TBI-related changes in personality and disorders of affect. ${ }^{36}$ A classic example involves the well-known 19th century case of Phineas Gage, who miraculously survived a severe TBI to the left frontal lobe only to develop personality changes which prompted those who knew him to declare that he was "no longer Gage". 37

In an insightful longitudinal study, Mayer et $\mathrm{al}^{38}$ investigated the temporal dynamics associated with the neural correlates of cognitive and emotional dysfunction across the first 6 months following TBI. The motivation of the study lay in the current difficulty to identify TBI patients at an early stage, who then fail to recover their cognitive acuity and emotional well-being. In a group of 27 mild TBI patients and 26 healthy control subjects, the researchers investigated spontaneous fluctuations in the DMN and in frontoparietal task-related networks as measured using fMRI. Despite the absence of positive findings on $T_{1}$ and $T_{2}$ scans acquired from these patients, significant differences in self-reported cognitive, emotional, and somatic function were identified in the TBI group. This may indicate that DTI tractography and white matter connectivity analysis should be undertaken in patients who suffer from personality changes, particularly when patients have negative findings on structural $T_{1}$ and $\mathrm{T}_{2}$ scans.

In a review of mild TBI pathophysiology in the pediatric population, Choe et $\mathrm{al}^{39}$ acknowledged that, although postconcussion symptoms are typically short-lived, longerlasting deficits can be particularly disruptive to the developing brain, and may result in changes of personality and in psychiatric complications later in life. Concussions do not typically result in structural damage which is visible on conventional computed tomography or MRI, whereas advanced neuroimaging modalities such as DTI and diffusion spectrum imaging reveal both microstructural as well as functional neurobiological changes, including DAI, metabolic impairment, neural activation changes, and cerebral blood flow perturbations which can contribute to acute symptomatology. Although such functional changes generally recover to baseline within approximately 1 week postinjury, sustaining recurrent injuries before full recovery was identified as a factor which can increase the potential for chronic deficits such as personality changes and mental disease.

Because mild TBI can be difficult to identify using standard MRI methods, functional studies should be prioritized for this large subpopulation. For example, in addition to fMRI, the use of electrophysiological techniques such as EEG and MEG has led to promising results in identifying physiological correlates of personality change, affective lability, and depression symptoms, as illustrated in a recent study by Huang et al. ${ }^{40}$ These authors studied both blast- and non-blast-related TBI patients and found that MEG slow wave generation in prefrontal areas is positively correlated with personality changes. The overall findings of the study suggest that MEG may be capable of detecting the effects of DAI-related deafferentation as well as of abnormal cholinergic signaling after injury, which may support MEG as a tool for assisting the diagnosis of mild TBI.

In military personnel returning from conflicts overseas, posttraumatic stress disorder, clinical depression, and TBI are often comorbid. In addition, collegiate and professional athletes involved in contact sports are more likely to suffer from multiple concussions compared to the general population. ${ }^{41}$ Studies have shown that such traumatic events increase the risk for chronic traumatic encephalopathy, a potentially serious neurodegenerative condition whose diagnosis can be attempted only postmortem based on currently available technology. ${ }^{42}$ American National Football League players such as Jovan Belcher (Kansas City Chiefs, age at suicide: 25), Chris Henry (Cincinnati Bengals, age at death: 26), Justin Strzelczyk (Pittsburgh Steelers, age at suicide: 36), and many others were diagnosed with chronic traumatic encephalopathy in a postmortem study of athletes who had exhibited symptoms of this condition during their lives. ${ }^{43}$ Simmons and Matthews ${ }^{44}$ reviewed the neural circuitry associated with posttraumatic stress disorder both in the presence as well as absence of mild TBI in an effort to distinguish between the distinct effects of these two conditions upon the brain. They suggested that the middle frontal gyrus is implicated in both conditions, with additional involvement of the dorsolateral prefrontal cortex, orbital cortex, and hippocampus. Nevertheless, whereas the middle frontal gyrus is overactivated in posttraumatic stress disorder, this structure is underactivated in TBI patients, which suggests that additional research is warranted to understand the role of the middle frontal gyrus in mediating posttraumatic stress disorder in the presence of TBI.

Clinical depression is frequently comorbid with TBI, although it is not well established whether its presence is 
due to the neurochemical and structural changes prompted by injury or rather to the emotional burden of disability. ${ }^{45}$ Motivated by evidence that hippocampal dysfunction plays a role in the etiology of mood disorders, Jorge et $\mathrm{al}^{46}$ imaged 37 TBI patients using MRI and found a significant statistical interaction between the presence of TBI and that of mood disorders, as well as a significant inverse correlation between hippocampal volumes and mood disturbance severity. Unfortunately, an insufficient amount of functional neuroimaging research like this has been carried out, despite repeated recommendations such as those of Vaishnavi et $\mathrm{al}^{47}$ who pointed out the scarcity of empirical data which can guide psychiatric treatment of TBI patients.

\section{Clinical utility of TBI functional neuroimaging}

Based on literature discussed in this review, we identify five major clinical goals whose achievement can greatly benefit from functional neuroimaging studies. As listed in the Table 1, these are:

1. Distinguishing between vegetative, minimally-conscious and locked-in patients

2. Designing novel interventions to reduce motor deficits after TBI

3. Determining how clinical care can be improved to alleviate memory deficits

4. Identifying how rehabilitation can minimize loss of cognitive control after brain injury

5. Formulating clinical strategies to alleviate personality changes and mental health sequelae.

To assist the reader in summarizing our findings, Table 1 also lists the major findings and conclusions of the studies discussed. In the case of the first clinical goal, determining whether a patient is in a vegetative, minimally-conscious, or locked-in state is of tremendous clinical utility for establishing basic communication with patients who appear to be unresponsive and for formulating clinical care appropriately. ${ }^{48}$ In the case of the second goal, reducing motor deficits in TBI patients is very important because it can help TBI survivors resume normal day-to-day activities and minimize caregiver assistance. Alleviating memory deficits and reversing the deterioration of cognitive control are also important goals of rehabilitation, partly because improvements in life quality, the resumption of gainful employment, and other aims often require substantial recovery of intellectual capacity. Finally, alleviating personality changes and mental health sequelae can allow TBI survivors to reintegrate into society, achieve and maintain emotional stability, and avoid social conflicts.

\section{Conclusion}

The challenges of understanding what happens to the human brain after traumatic injury stem not only from the inherent complexity of brain function, but also from the patient-topatient variability in injury types and in the neural, metabolic, and hemodynamic responses to them. In this review, we have attempted to highlight some of the most important trends in TBI functional neuroimaging and to provide suggestions that can help in translating the findings of neuroimaging studies into clinically useful information. To assist in the clinical care of patients with prolonged loss of consciousness, more functional studies are needed to distinguish between minimally-conscious patients and those in a vegetative state, and also to understand how minimal consciousness and the vegetative state influence the duration and extent of posttraumatic amnesia. Such studies should preferably be multimodal and should include at least one modality which offers insight on brain metabolism (eg, PET) and one modality which provides a measure of blood oxygenation (eg, BOLD fMRI). This is because the relationship between glucose metabolism and oxygen consumption is complex enough to make inferences of metabolic dysfunction based on blood oxygenation levels - or vice versa - which is challenging and frequently unreliable.

Of all the functional domains discussed in this review, the area of personality changes prompted by TBI appears to have received the least amount of attention from functional neuroimaging researchers. A detailed scientific literature search focused on articles covering this topic and published in the past 5 years has yielded surprisingly few studies which used functional neuroimaging to study personality changes after TBI. This paucity of research is both surprising and troubling, particularly given that personality changes are quite frequent following TBIs of all severities. ${ }^{49}$ Though psychometric studies of posttraumatic personality change abound in the recent literature, ${ }^{50-52}$ the relationship between psychometric measures and functional neuroimaging metrics remains almost entirely unexplored. This indicates the existence of a significant knowledge gap on TBI, which should be promptly addressed by future studies.

Although many of the studies discussed have identified functional relationships between distinct brain regions which exhibit abnormal function after TBI, these findings are difficult to generalize across the TBI population due to its heterogeneity as well as the great variety of functional protocols 


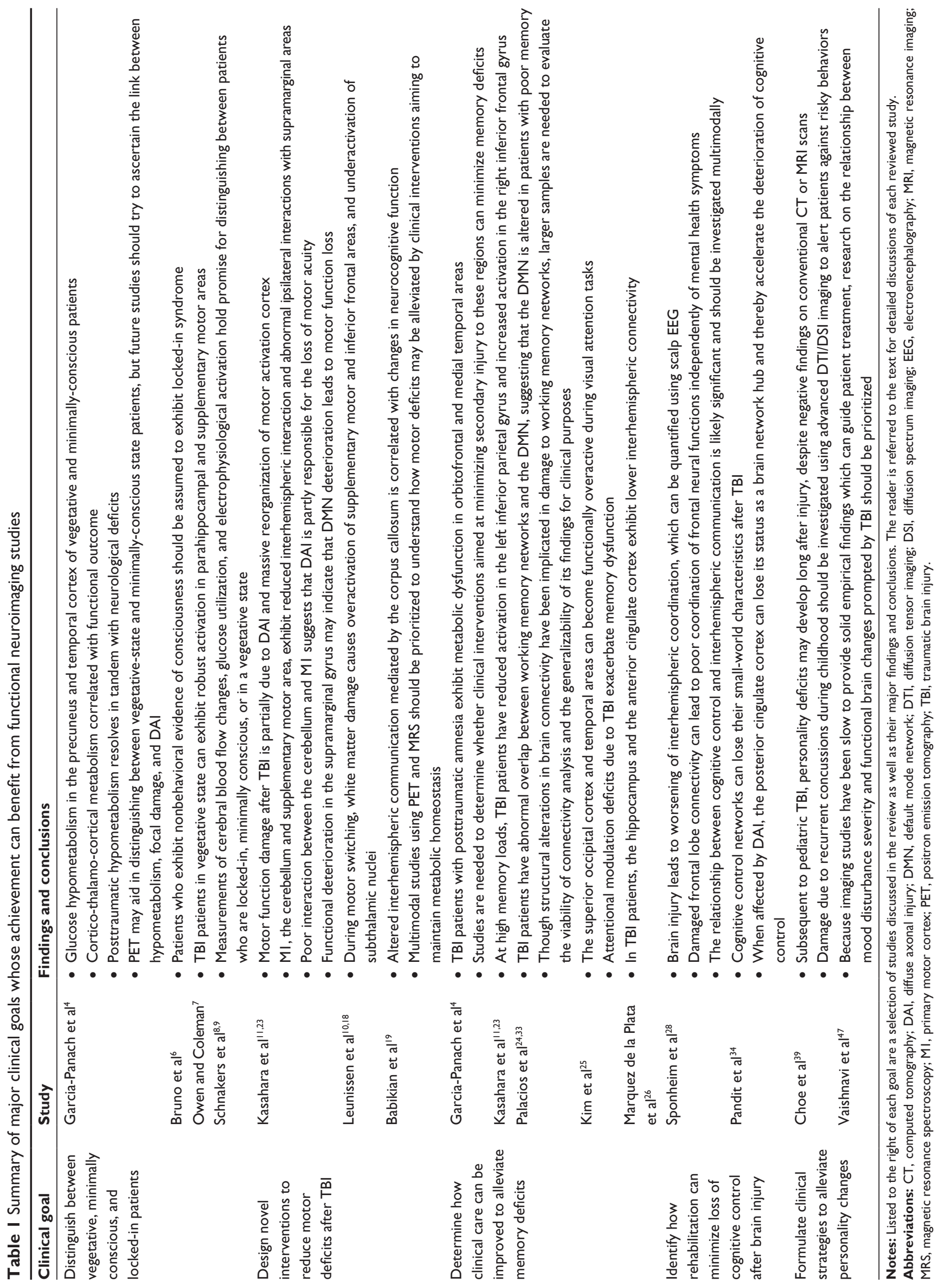


used in different experiments. For this reason, greater sample sizes are needed (particularly in fMRI studies) to account for the statistical variability of BOLD measurements across subjects and brain areas. In addition, experimental protocols involving tasks should be standardized to facilitate meta-analyses and large population studies. We advocate the creation of an international repository of functional TBI neuroimaging data which can allow researchers from across the world to pool and analyze not only resting-state data from distinct study sites, but also data which involves eventrelated brain activations. Such a database would facilitate the study of specific domains of brain function (eg, motor ability, attention, memory, cognitive control, etc) in an effort to gain deeper understanding of how TBI affects brain function domains differently and how such understanding can be translated into clinically useful information.

\section{Disclosure}

The authors report no conflicts of interest in this work.

\section{References}

1. Irimia A, Wang B, Aylward SR, et al. Neuroimaging of structural pathology and connectomics in traumatic brain injury: toward personalized outcome prediction. Neuroimage Clin. 2012;1(1):1-17.

2. Irimia A, Chambers MC, Alger JR, et al. Comparison of acute and chronic traumatic brain injury using semi-automatic multimodal segmentation of MR volumes. J Neurotrauma. 2011;28(11):2287-2306.

3. Hunter JV, Wilde EA, Tong KA, Holshouser BA. Emerging imaging tools for use with traumatic brain injury research. J Neurotrauma. 2012; 29(4):654-671.

4. Garcia-Panach J, Lull N, Lull JJ, et al. A voxel-based analysis of FDG-PET in traumatic brain injury: regional metabolism and relationship between the thalamus and cortical areas. J Neurotrauma. 2011; 28(9):1707-1717.

5. Zhang DY, Snyder AZ, Fox MD, Sansbury MW, Shimony JS, Raichle ME. Intrinsic functional relations between human cerebral cortex and thalamus. J Neurophysiol. 2008;100(4):1740-1748.

6. Bruno MA, Vanhaudenhuyse A, Thibaut A, Moonen G, Laureys S. From unresponsive wakefulness to minimally conscious PLUS and functional locked-in syndromes: recent advances in our understanding of disorders of consciousness. J Neurol. 2011;258(7):1373-1384.

7. Owen AM, Coleman MR. Detecting awareness in the vegetative state. Ann N Y Acad Sci. 2008;1129:130-138.

8. Schnakers C, Perrin F, Schabus M, et al. Voluntary brain processing in disorders of consciousness. Neurology. 2008;71(20):1614-1620.

9. Schnakers C, Perrin F, Schabus M, et al. Detecting consciousness in a total locked-in syndrome: an active event-related paradigm. Neurocase. 2009;15(4):271-277.

10. Leunissen I, Coxon JP, Caeyenberghs K, Michiels K, Sunaert S, Swinnen SP. Task switching in traumatic brain injury relates to corticosubcortical integrity. Hum Brain Mapp. 2014;35(5):2459-2469.

11. Kasahara M, Menon DK, Salmond CH, et al. Altered functional connectivity in the motor network after traumatic brain injury. Neurology. 2010; 75(2):168-176.

12. Buckner RL, Andrews-Hanna JR, Schacter DL. The brain's default network: anatomy, function, and relevance to disease. Ann N Y Acad Sci. 2008;1124:1-38.

13. van den Heuvel MP, Sporns O. Rich-club organization of the human connectome. J Neurosci. 2011;31(44):15775-15786.
14. Irimia A, Van Horn JD. Systematic network lesioning reveals the core white matter scaffold of the human brain. Front Hum Neurosci. 2014; 8:51.

15. Reed CL, Caselli RJ. The nature of tactile agnosia: a case study. Neuropsychologia. 1994;32(5):527-539.

16. Rushworth MF, Krams M, Passingham RE. The attentional role of the left parietal cortex: the distinct lateralization and localization of motor attention in the human brain. J Cogn Neurosci. 2001;13(5):698-710.

17. Irimia A, Chambers MC, Torgerson CM, et al. Patient-tailored connectomics visualization for the assessment of white matter atrophy in traumatic brain injury. Front Neurol. 2012;3:10.

18. Leunissen I, Coxon JP, Geurts M, et al. Disturbed cortico-subcortical interactions during motor task switching in traumatic brain injury. Hum Brain Mapp. 2013;34(6):1254-1271.

19. Babikian T, Marion SD, Copeland S, et al. Metabolic levels in the corpus callosum and their structural and behavioral correlates after moderate to severe pediatric TBI. J Neurotrauma. 2010;27(3):473-481.

20. Nugent AC, Martinez A, D'Alfonso A, Zarate CA, Theodore WH. The relationship between glucose metabolism, resting-state fMRI BOLD signal, and GABA(A)-binding potential: a preliminary study in healthy subjects and those with temporal lobe epilepsy. J Cereb Blood Flow Metab. 2015;35(4):583-591.

21. McAllister TW. Neurobehavioral sequelae of traumatic brain injury: evaluation and management. World Psychiatry. 2008;7(1):3-10.

22. Scimeca JM, Badre D. Striatal contributions to declarative memory retrieval. Neuron. 2012;75(3):380-392.

23. Kasahara M, Menon DK, Salmond $\mathrm{CH}$, et al. Traumatic brain injury alters the functional brain network mediating working memory. Brain Inj. 2011;25(12):1170-1187.

24. Palacios EM, Sala-Llonch R, Junque C, et al. White matter integrity related to functional working memory networks in traumatic brain injury. Neurology. 2012;78(12):852-860.

25. Kim J, Whyte J, Patel S, et al. A perfusion fMRI study of the neural correlates of sustained-attention and working-memory deficits in chronic traumatic brain injury. Neurorehabil Neural Repair. 2012; 26(7):870-880.

26. Marquez de la Plata CD, Garces J, Shokri Kojori E, et al. Deficits in functional connectivity of hippocampal and frontal lobe circuits after traumatic axonal injury. Arch Neurol. 2011;68(1):74-84.

27. Scheibel RS, Newsome MR, Troyanskaya M, et al. Altered brain activation in military personnel with one or more traumatic brain injuries following blast. J Int Neuropsychol Soc. 2012;18(1):89-100.

28. Sponheim SR, McGuire KA, Kang SS, et al. Evidence of disrupted functional connectivity in the brain after combat-related blast injury. Neuroimage. 2011;54(Suppl 1):S21-S29.

29. Duncan CC, Summers AC, Perla EJ, Coburn KL, Mirsky AF. Evaluation of traumatic brain injury: brain potentials in diagnosis, function, and prognosis. Int J Psychophysiol. 2011;82(1):24-40.

30. Alexopoulos GS, Hoptman MJ, Kanellopoulos D, Murphy CF, Lim KO, Gunning FM. Functional connectivity in the cognitive control network and the default mode network in late-life depression. J Affect Disord. 2012; 139(1):56-65.

31. Irimia A, Van Horn JD, Halgren E. Source cancellation profiles of electroencephalography and magnetoencephalography. Neuroimage. 2012;59(3):2464-2474.

32. Irimia A, Erhart MJ, Brown TT. Variability of magnetoencephalographic sensor sensitivity measures as a function of age, brain volume and cortical area. Clin Neurophysiol. 2014;125(10):1973-1984.

33. Palacios EM, Sala-Llonch R, Junque C, et al. Resting-state functional magnetic resonance imaging activity and connectivity and cognitive outcome in traumatic brain injury. JAMA Neurol. 2013;70(7): 845-851.

34. Pandit AS, Expert P, Lambiotte R, et al. Traumatic brain injury impairs small-world topology. Neurology. 2013;80(20):1826-1833.

35. Wilde EA, Newsome MR, Bigler ED, et al. Brain imaging correlates of verbal working memory in children following traumatic brain injury. Int J Psychophysiol. 2011;82(1):86-96. 
36. Schwarzbold M, Diaz A, Martins ET, et al. Psychiatric disorders and traumatic brain injury. Neuropsychiatr Dis Treat. 2008;4(4):797-816.

37. Van Horn JD, Irimia A, Torgerson CM, Chambers MC, Kikinis R, Toga AW. Mapping connectivity damage in the case of Phineas Gage. PLoS One. 2012;7(5):e37454.

38. Mayer AR, Mannell MV, Ling J, Gasparovic C, Yeo RA. Functional connectivity in mild traumatic brain injury. Hum Brain Mapp. 2011; 32(11):1825-1835.

39. Choe MC, Babikian T, DiFiori J, Hovda DA, Giza CC. A pediatric perspective on concussion pathophysiology. Curr Opin Pediatr. 2012; 24(6):689-695.

40. Huang MX, Nichols S, Baker DG, et al. Single-subject-based wholebrain MEG slow-wave imaging approach for detecting abnormality in patients with mild traumatic brain injury. Neuroimage Clin. 2014;5 109-119.

41. Covassin T, Elbin R, Kontos A, Larson E. Investigating baseline neurocognitive performance between male and female athletes with a history of multiple concussion. J Neurol Neurosurg Psychiatry. 2010; 81(6):597-601.

42. Baugh CM, Robbins CA, Stern RA, McKee AC. Current understanding of chronic traumatic encephalopathy. Curr Treat Options Neurol. 2014; 16(9):306.

43. McKee AC, Cantu RC, Nowinski CJ, et al. Chronic traumatic encephalopathy in athletes: progressive tauopathy after repetitive head injury. J Neuropathol Exp Neurol. 2009;68(7):709-735.

44. Simmons AN, Matthews SC. Neural circuitry of PTSD with or without mild traumatic brain injury: a meta-analysis. Neuropharmacology. 2012;62(2):598-606.
45. van Reekum R, Cohen T, Wong J. Can traumatic brain injury cause psychiatric disorders? J Neuropsychiatry Clin Neurosci. 2000;12(3): 316-327.

46. Jorge RE, Acion L, Starkstein SE, Magnotta V. Hippocampal volume and mood disorders after traumatic brain injury. Biol Psychiatry. 2007; 62(4):332-338.

47. Vaishnavi S, Rao V, Fann JR. Neuropsychiatric problems after traumatic brain injury: unraveling the silent epidemic. Psychosomatics. 2009; 50(3):198-205.

48. Monti MM, Vanhaudenhuyse A, Coleman MR, et al. Willful modulation of brain activity in disorders of consciousness. N Engl J Med. 2010; 362(7):579-589.

49. Vasa RA, Suskauer SJ, Thorn JM, et al. Prevalence and predictors of affective lability after paediatric traumatic brain injury. Brain Inj. 2015; 29(7-8):921-928.

50. Trenchard SO, Rust S, Bunton P. A systematic review of psychosocial outcomes within 2 years of paediatric traumatic brain injury in a schoolaged population. Brain Inj. 2013;27(11):1217-1237.

51. Li L, Liu J. The effect of pediatric traumatic brain injury on behavioral outcomes: a systematic review. Dev Med Child Neurol. 2013;55(1): $37-45$.

52. Mendez MF, Owens EM, Jimenez EE, Peppers D, Licht EA. Changes in personality after mild traumatic brain injury from primary blast vs blunt forces. Brain Inj. 2013;27(1):10-18.
Neuropsychiatric Disease and Treatment

\section{Publish your work in this journal}

Neuropsychiatric Disease and Treatment is an international, peerreviewed journal of clinical therapeutics and pharmacology focusing on concise rapid reporting of clinical or pre-clinical studies on a range of neuropsychiatric and neurological disorders. This journal is indexed on PubMed Central, the 'PsycINFO' database and CAS,

\section{Dovepress}

and is the official journal of The International Neuropsychiatric Association (INA). The manuscript management system is completely online and includes a very quick and fair peer-review system, which is all easy to use. Visit http://www.dovepress.com/testimonials.php to read real quotes from published authors. 